\title{
Treatment Options for Dementia with Lewy Bodies: A Network Meta-Analysis of Randomised Control Trials
}

\author{
Amir A. Tahami Monfared - Mitesh Desai - Robert Hughes • \\ Stefano Lucherini · Yunni Yi · Richard Perry
}

Received: April 3, 2020 / Published online: June 3, 2020

(C) The Author(s) 2020

\begin{abstract}
Introduction: Dementia with Lewy bodies (DLB) is the third most common type of dementia after Alzheimer's disease (AD) and vascular dementia. Treatment is targeted at specific disease manifestations/symptoms. While donepezil is approved for the treatment of DLB in Japan, to date no other treatment has been approved for this indication anywhere in the world. Notwithstanding, many of the medications that are approved for $\mathrm{AD}$ are widely used in the treatment of DLB with varying degrees of success. Consequently, clinical evidence is limited, and there is a need to understand the comparative efficacy and safety of currently used therapies for DLB. The aim of
\end{abstract}

Digital Features To view digital features for this article go to https://doi.org/10.6084/m9.figshare.12349778.

Electronic Supplementary Material The online version of this article (https://doi.org/10.1007/s40120020-00198-0) contains supplementary material, which is available to authorized users.

A. A. Tahami Monfared ( $\square)$

Eisai Inc., Woodcliff Lake, NJ, USA

e-mail: amir_tahami@eisai.com

\section{A. A. Tahami Monfared}

Epidemiology, Biostatistics, and Occupational

Health, McGill University, Montreal, QC, Canada

M. Desai · R. Hughes · S. Lucherini · Y. Yi · R. Perry Adelphi Values, Bollington, UK this study was to conduct a network metaanalysis (NMA) to evaluate the outcomes of the available treatment options based on currently used trial endpoints.

Methods: Using data from a previously published systematic review, we conducted an NMA to investigate the efficacy and safety of treatments in patients with DLB. Networks were based on change from baseline of efficacy endpoints (Mini-Mental State Examination; Neuropsychiatric Inventory; Unified Parkinson's Disease Rating Scale) and rate of safety events (overall adverse events [AEs]; discontinuations; discontinuations due to AEs; psychiatric events).

Results: Focused around a common treatment option of placebo, the NMA comprised studies on donepezil, rivastigmine, memantine and quetiapine. Donepezil $3 \mathrm{mg}, 5 \mathrm{mg}$ and $10 \mathrm{mg}$ doses were compared against each other and placebo. Overall, donepezil consistently performed better than the alternative treatments when compared to placebo for all efficacy and safety endpoints. However, the small sample size and/or heterogeneity of the studies led to uncertainty, resulting in no statistically significant differences favouring any treatment above another or placebo.

Conclusion: Despite the lack of statistical significance, when assessing the efficacy and safety outcomes for each drug in the evidence network, donepezil appeared to have a more favourable overall benefit/risk profile for 
patients with DLB. Further comparative trials are required to improve understanding of the true difference between existing and potential future treatment options.

Keywords: Dementia with Lewy bodies; Donepezil; Memantine; Mini-Mental State Examination; Network meta-analysis; Neuropsychiatric Inventory; Quetiapine; Rivastigmine; Unified Parkinson's Disease Rating Scale

\section{Key Summary Points}

\section{Why carry out this study?}

Dementia with Lewy bodies (DLB) is the third most common type of dementia after Alzheimer's Disease and vascular dementia; however, very few comparative trials have been conducted between the available therapy options.

Currently, donepezil is the only treatment specifically approved for the treatment of DLB, although many treatments approved for Alzheimer's disease are also used, leading to poorly defined treatment guidelines.

A network meta-analysis was conducted to produce comparative evidence between the available treatment options for DLB.

\section{What was learned from the study?}

While very few results produced were statistically significant, the trend seen suggests that donepezil has a more favourable overall benefit/risk profile for patients with DLB.

Although the conclusions were supported by consistent trends across all included outcome measures, the lack of significant results suggested that additional clinical trials would be required.

Additionally, the variability across patient groups within each trial led to significant heterogeneity between the different studies included in the analysis.

\section{INTRODUCTION}

Dementia with Lewy bodies (DLB) is the third most common type of dementia, after Alzheimer's Disease (AD) and vascular dementia, accounting for approximately $10-15 \%$ of all dementia cases [1]. It is estimated that 1.3 million individuals in the USA and 100,000 individuals in the UK are affected by DLB [2].

DLB is a gradually progressive condition occurring as a result of intraneuronal inclusions-consisting of $\alpha$-synuclein aggregates and ubiquitin (known as Lewy bodies) - in the neocortex, forebrain, brainstem and other parts of the nervous system [3]. Individuals suffer worsening cognitive, neuropsychiatric, autonomic and motor impairment [4]. The decline in function results in a high economic burden to society, with increasing direct and indirect costs, especially following admission of the individual into nursing homes [5-7].

Treatment is targeted at specific disease manifestations or symptoms [8]. Based on the results from two randomised controlled trials (RCTs), which demonstrated improved cognitive function in patients with DLB treated with Eisai's ARICEPT ${ }^{\circledR}$ (donepezil) [9], ARICEPT was approved by the Japanese Pharmaceuticals and Medical Devices Agency in 2014 to become the world's first and currently only licensed treatment for DLB [10]. Worldwide, drugs licensed for the treatment of $\mathrm{AD}$ are widely used to treat patients with DLB, with varying degrees of success $[4,8,11,12]$. Given the resulting limited available clinical evidence, the objective of this study was to conduct a network meta-analysis (NMA) to explore the comparative efficacy and safety of current pharmacological interventions used to treat patients with DLB, based on available evidence from RCTs.

NMAs are used widely for health technology appraisals and regulatory approval discussions where direct trials have not been conducted between treatments of interest. Increasingly, they are being performed to inform decisionmaking regarding the comparative efficacy and safety of alternative treatments. The NMA is an extension of traditional meta-analysis 
approaches that utilises all available evidence to provide an assessment of the indirect comparison of treatments, where direct evidence, due to a lack of head-to-head studies, is unavailable.

\section{METHODS}

The NMA was conducted based on the findings of a previously published systematic literature review of RCTs reporting on pharmacological interventions in DLB [13]. Prior to conducting the NMA, a feasibility assessment was carried out to confirm that indirect comparisons between DLB pharmacological interventions were viable for the proposed outcome measures and that there were no new data that may impact the NMA expected to be published in the immediate future. The protocol for the analysis was developed in consultation with the Preferred Reporting Items for Systematic Reviews and Meta-Analysis Protocols (PRISMAP) Statement $[14,15]$. This article is based on previously conducted studies and does not contain any studies with human participants or animals performed by any of the authors.

The aim of this study was to determine the comparative clinical efficacy of cognitive-behavioural treatments that are commonly used in patients with DLB. It is understood that some patients may have received L-dopa, dopamine agonists, antidepressants or benzodiazepines at baseline. However, the proportion of such medications was assumed to be the same within clinical studies, as this was unreported in most studies. Additionally, evidence has shown that that L-dopa does not compromise the cognitive or behavioural profiles of patients with DLB either acutely or over a longer period of time, meaning they will have a limited impact on the included outcome measures [16].

Within the available studies, donepezil was the only treatment that explicitly compared the outcomes for the different dose amounts. All other studies focusing on alternative therapies included a range of dose amount within a single arm due to the titration process.

Donepezil has been approved globally for the treatment of dementia of the Alzheimer's type, with the $5 \mathrm{mg}$ and $10 \mathrm{mg}$ dosages being approved in the USA and Europe. In Japan specifically, donepezil dose options of 3, 5 and $10 \mathrm{mg}$ have been approved for the treatment of both $\mathrm{AD}$ and DLB [10, 17]. On this basis, the donepezil $3 \mathrm{mg}$ dose was included as a specific treatment comparator within the NMA. Due to citalopram and risperidone forming a disconnected network, these two medications were excluded from the analysis.

A unified scale to comprehensively assess the symptoms or disease progression of DLB is currently lacking. Therefore, a number of different measures were included as efficacy outcome measures in the analysis, with the most most commonly reported ones being the Mini-Mental State Examination (MMSE) [11, 12, 18-21], variations of the Neuropsychiatric Inventory (NPI) instrument $[11,12,18,21-24]$ and variations of the Unified Parkinson's Disease Rating Scale (UPDRS) [25]. While these measures may not capture the full range of effects for each of the included treatment options, they were selected as the most consistently reported measures across the different studies available for patients with DLB.

A number of variations of the NPI instrument are used across different studies. However, the ten-item NPI (NPI-10) was selected for inclusion in the base-case NMA analysis, as this version was the most commonly reported instrument across the available trials. Most of the identified studies that reported the UPDRS measure only included part III of the instrument, which focuses on the motor functions of individuals, rather than using the complete UPDRS instrument.

Additionally, a range of safety measures were included to assess various aspects of the safety profiles. These measures were the overall rate of adverse events (AEs), discontinuation rate, discontinuations due to AEs and the rate of psychiatric events. A summary of all outcome measures are included in Appendix 1 in the Electronic Supplementary Material (ESM).

Variations in the time horizon evaluated, the baseline scores for outcome measures and the patient populations included within each trial were taken into consideration. These variations are likely to have introduced higher levels of heterogeneity between the studies than that 


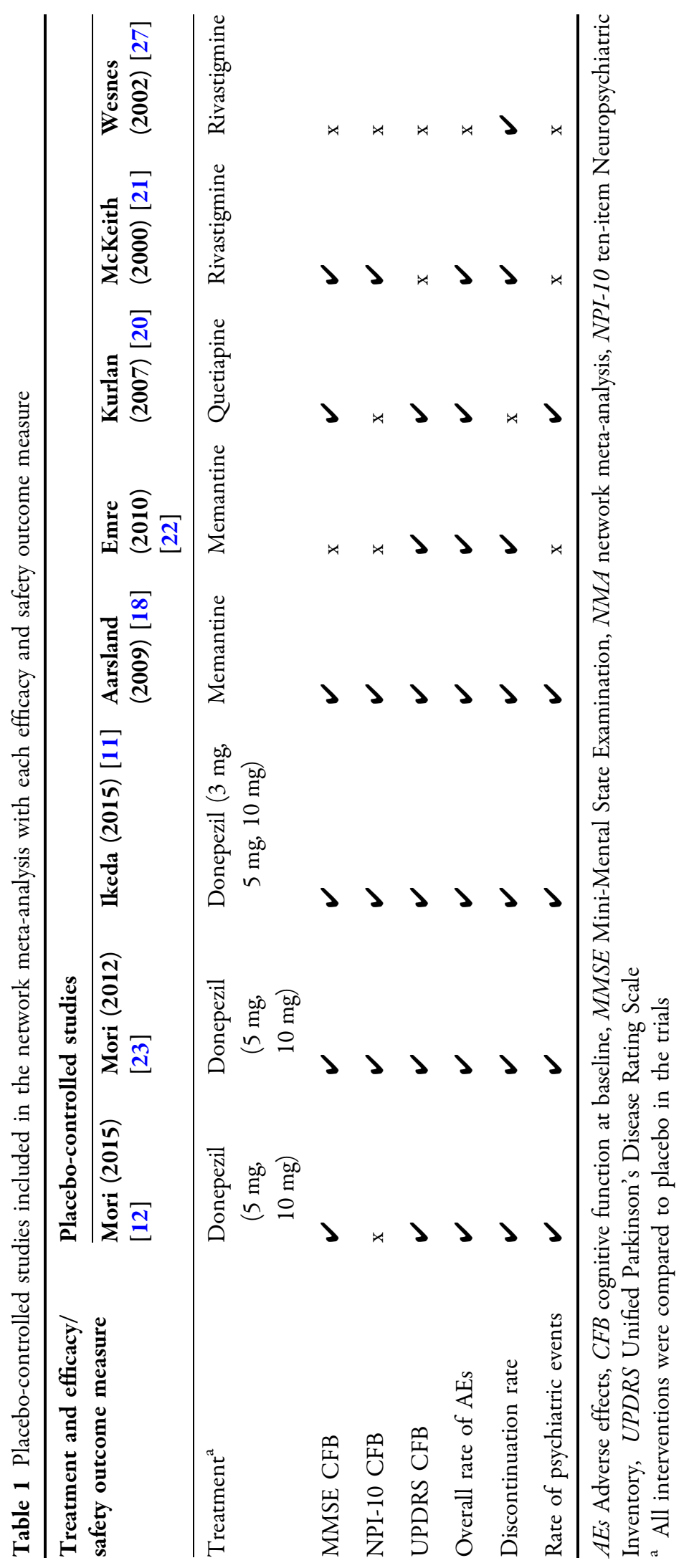


expected between any studies conducted independently. The variations observed in the time horizons and the baseline scores were deemed sufficiently limited to allow the results to be meaningful. Furthermore, given the number of trials available, excluding trials with significantly differing characteristics would introduce a greater bias than the effect of including the studies in the analysis. The variation in the baseline patient populations was considered to have the potential to significantly impact treatment outcomes. Therefore, it was decided to exclude trials with non-DLB specific patient populations as part of the sensitivity analyses conducted. The NMAs were performed using Bayesian methods, following the guidance provided in the National Institute for Health and Clinical Excellence (NICE) Decision Support

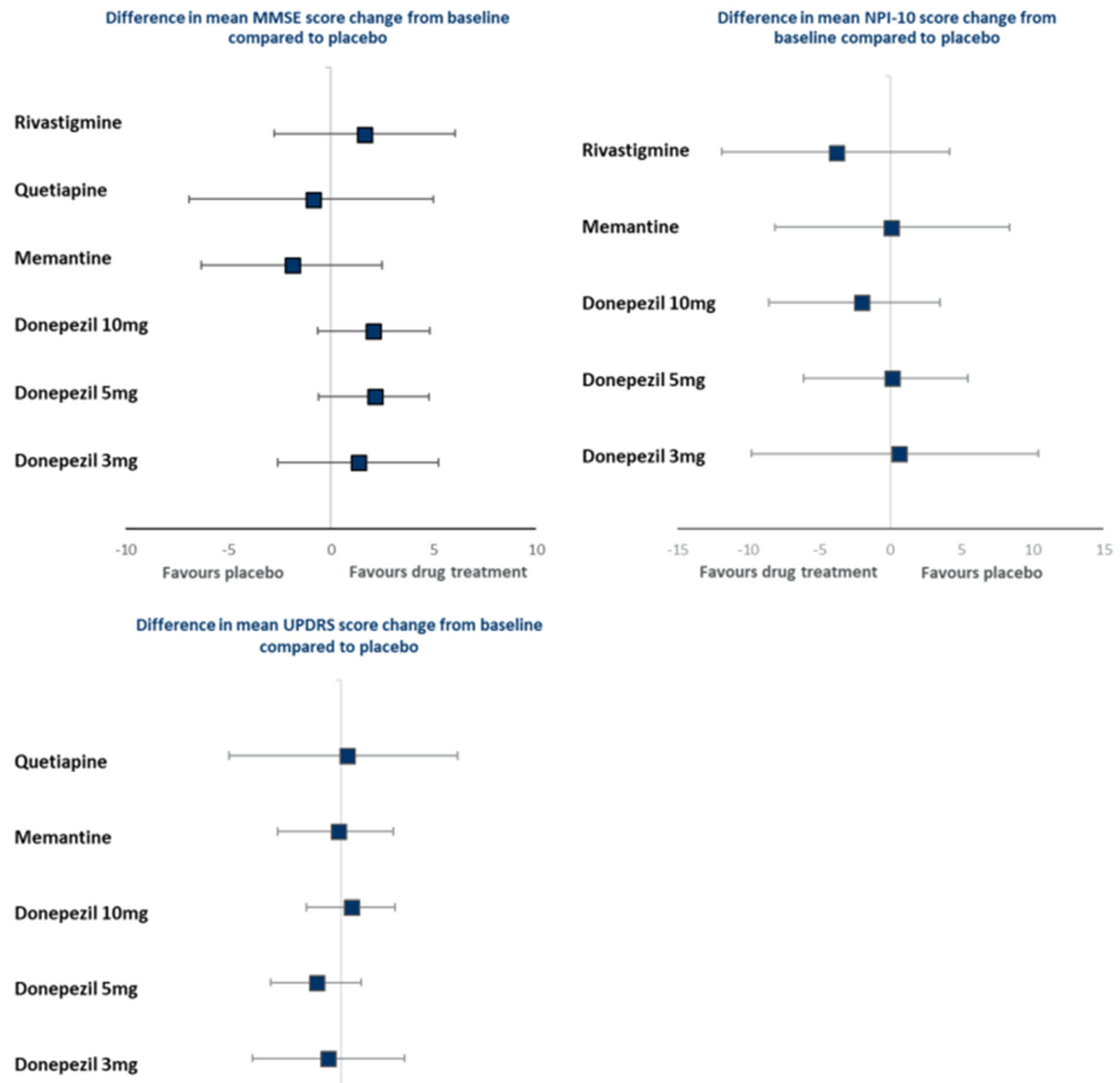

Fig. 1 Forest plots for efficacy outcome measures. MMSE Mini-Mental State Examination, NPI-10 ten-item Neuropsychiatric Inventory, UPDRS Unified Parkinson's Disease Rating Scale 
Table 2 Likelihood of each treatment option being the most effective and the expected rank of each treatment in the data set

\begin{tabular}{|c|c|c|c|c|}
\hline \multirow[t]{2}{*}{$\begin{array}{l}\text { Efficacy outcome measures } \\
\text { of treatment }\end{array}$} & \multicolumn{2}{|c|}{$\begin{array}{l}\text { Probability } \\
\text { of being the } \\
\text { most } \\
\text { effective } \\
\text { treatment }\end{array}$} & \multicolumn{2}{|c|}{$\begin{array}{l}\text { Rank of } \\
\text { treatment }\end{array}$} \\
\hline & Mean & SD & Mean & SD \\
\hline \multicolumn{5}{|l|}{ MMSE } \\
\hline Placebo & 0 & 0.03 & 5.06 & 0.9 \\
\hline Donepezil $3 \mathrm{mg}$ & 0.14 & 0.35 & 3.46 & 1.61 \\
\hline Donepezil $5 \mathrm{mg}$ & 0.26 & 0.44 & 2.42 & 1.24 \\
\hline Donepezil $10 \mathrm{mg}$ & 0.23 & 0.42 & 2.52 & 1.23 \\
\hline Memantine & 0.01 & 0.12 & 6.18 & 1.28 \\
\hline Quetiapine & 0.09 & 0.28 & 5.21 & 1.95 \\
\hline Rivastigmine & 0.26 & 0.44 & 3.15 & 1.77 \\
\hline \multicolumn{5}{|l|}{ NPI-10 } \\
\hline Placebo & 0.01 & 0.1 & 4.04 & 1.17 \\
\hline Donepezil $3 \mathrm{mg}$ & 0.13 & 0.34 & 4.11 & 1.86 \\
\hline Donepezil $5 \mathrm{mg}$ & 0.03 & 0.18 & 4.05 & 1.34 \\
\hline Donepezil $10 \mathrm{mg}$ & 0.19 & 0.39 & 2.7 & 1.32 \\
\hline Memantine & 0.13 & 0.33 & 3.92 & 1.8 \\
\hline Rivastigmine & 0.5 & 0.5 & 2.19 & 1.54 \\
\hline \multicolumn{5}{|l|}{ UPDRS } \\
\hline Placebo & 0.01 & 0.12 & 3.85 & 1.07 \\
\hline Donepezil $3 \mathrm{mg}$ & 0.26 & 0.44 & 2.99 & 1.72 \\
\hline Donepezil $5 \mathrm{mg}$ & 0.35 & 0.48 & 2.12 & 1.12 \\
\hline Donepezil $10 \mathrm{mg}$ & 0.01 & 0.11 & 4.59 & 1.25 \\
\hline Memantine & 0.13 & 0.34 & 3.57 & 1.63 \\
\hline Quetiapine & 0.24 & 0.42 & 3.89 & 2.07 \\
\hline
\end{tabular}

SD Standard deviation

Unit Technical Support Document series on evidence synthesis [26]. The results of the analyses were measured as absolute and relative effect (difference in mean change from baseline for continuous variable and odds ratio [OR] for binomial variables), the probability of being the most effective or safest treatment and the rank of the treatment by outcomes. The results were deemed to be statistically significant if the 95\% confidence interval (CI) did not cross the origin. All analyses were performed with WinBUGS version 1.4 .

Both a fixed effect (FE) and random effect (RE) model were run for each network. The base-case model was selected according to the goodness of fit. The alternative modelling approach was included as one of the sensitivity analyses conducted. All other sensitivity analyses for all outcome measures were conducted using the modelling approach selected for the base-case model. Sensitivity analyses tested a range of different scenarios: the exclusion of mixed study populations comprising patients with other forms of dementia, including $\mathrm{AD}$ and Parkinson's disease dementia; the inclusion of additional data; or the pooling of all doses of donepezil (see Appendix 2 in the ESM for further details). The pooling of the donepezil dose amounts was included as part of the sensitivity analysis to align the approaches taken within other studies, where a range of dose amounts were included within a single treatment arm.

\section{RESULTS}

\section{Basic Features of the NMA}

A total of eight placebo-controlled RCTs were included in this NMA. Based on the included outcomes, data were available to produce networks with up to seven different treatment options: donepezil $3 \mathrm{mg}$, donepezil $5 \mathrm{mg}$, donepezil $10 \mathrm{mg}$, memantine, quetiapine, rivastigmine and placebo. Table 1 summarises the studies included for each of the outcome measures.

For all outcome measures, the RE model showed the best fit and was selected for the base-case analysis. 


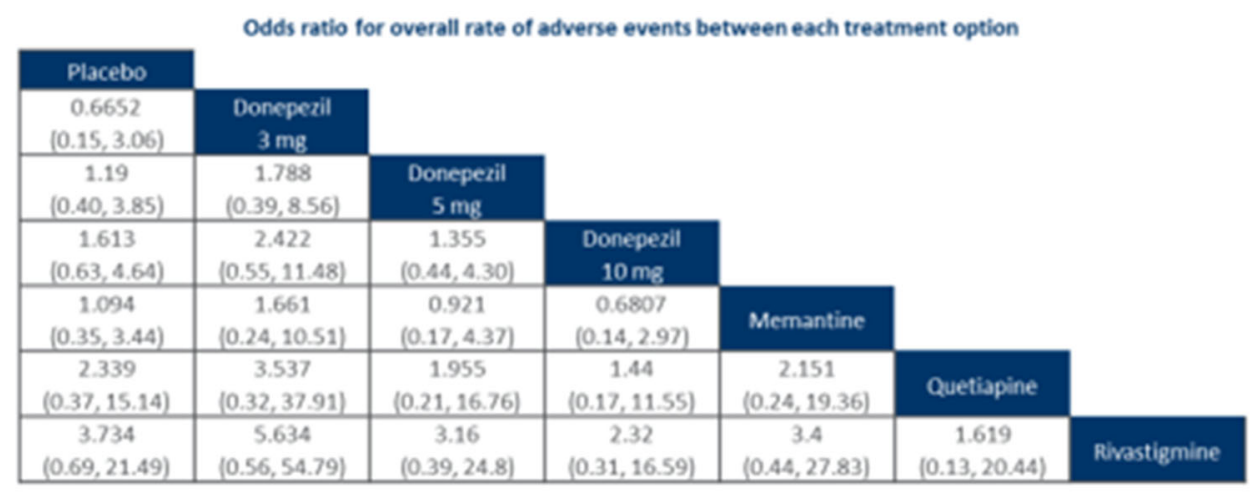

Odds ratio for rate of discontinuation between each treatment option

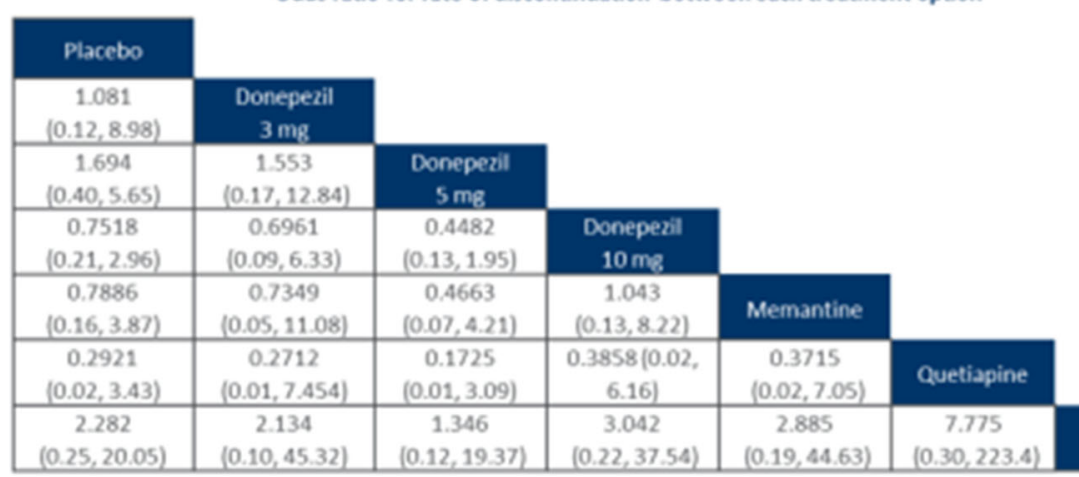

Rivastigmine

Odds ratio for rate of discontinuation due to AEs between each treatment option

\begin{tabular}{|c|c|c|c|c|c|c|}
\hline Placebo & & & & & & \\
\hline $\begin{array}{c}0.9096 \\
(0.04,18.25)\end{array}$ & $\begin{array}{c}\text { Donepezil } \\
3 \mathrm{mg}\end{array}$ & & & & & \\
\hline $\begin{array}{c}1.499 \\
(0.17,8.73)\end{array}$ & $\begin{array}{c}1.619 \\
(0.07,35.23)\end{array}$ & $\begin{array}{l}\text { Donepezil } \\
5 \mathrm{mg}\end{array}$ & & & & \\
\hline $\begin{array}{c}0.2821 \\
(0.03,1.97) \\
\end{array}$ & $\begin{array}{c}0.3068 \\
(0.01,6.90) \\
\end{array}$ & $\begin{array}{c}0.1901 \\
(0.02,1.58) \\
\end{array}$ & $\begin{array}{c}\text { Donepezil } \\
10 \mathrm{mg}\end{array}$ & & & \\
\hline $\begin{array}{c}0.8359 \\
(0.08,8.64)\end{array}$ & $\begin{array}{c}0.9207 \\
(0.02,49.65)\end{array}$ & $\begin{array}{c}0.556 \\
(0.03,14.49)\end{array}$ & $\begin{array}{c}2.949 \\
(0.15,79.70)\end{array}$ & Memantine & & \\
\hline $\begin{array}{c}0.85 \\
(0.00,901.8)\end{array}$ & $\begin{array}{c}0.9225 \\
(0.00,2035)\end{array}$ & $\begin{array}{c}0.5901 \\
(0.00,890.8)\end{array}$ & $\begin{array}{c}3.046 \\
(0.00,4915)\end{array}$ & $\begin{array}{c}1.031 \\
(0.00,1702]\end{array}$ & Quetiapine & \\
\hline $\begin{array}{c}1.04 \\
(0.04,27.71)\end{array}$ & $\begin{array}{c}1.155 \\
(0.01,115.5)\end{array}$ & $\begin{array}{c}0.688 \\
(0.02,39.6)\end{array}$ & $\begin{array}{c}3.697 \\
(0.09,209.8)\end{array}$ & $\begin{array}{c}1.254 \\
(0.021,69.9)\end{array}$ & $\begin{array}{c}1.2 \\
(0.00,4156)\end{array}$ & Rivastigmine \\
\hline
\end{tabular}

Odds ratio for rate of psychiatric events between each treatment option

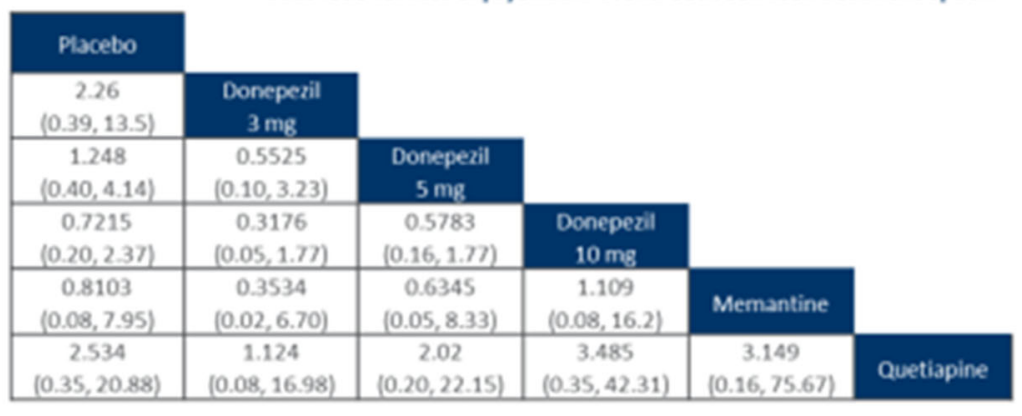

Fig. 2 Odds ratios for the safety outcome measures. Values in parenthesis are the 95\% confidence intervals. AEs Adverse events 
Table 3 Likelihood of each treatment option having the best safety outcome and the expected rank of each treatment in the data set

\begin{tabular}{|c|c|c|c|c|}
\hline \multirow[t]{2}{*}{$\begin{array}{l}\text { Treatment option/and } \\
\text { efficacy/safety outcome }\end{array}$} & \multicolumn{2}{|c|}{$\begin{array}{l}\text { Probability } \\
\text { of being the } \\
\text { safest } \\
\text { treatment }\end{array}$} & \multicolumn{2}{|c|}{$\begin{array}{l}\text { Rank of } \\
\text { treatment }\end{array}$} \\
\hline & Mean & SD & Mean & SD \\
\hline \multicolumn{5}{|l|}{ Overall AE rate } \\
\hline Placebo & 0.09 & 0.28 & 2.85 & 1.06 \\
\hline Donepezil $3 \mathrm{mg}$ & 0.59 & 0.49 & 1.98 & 1.48 \\
\hline Donepezil $5 \mathrm{mg}$ & 0.08 & 0.26 & 3.62 & 1.51 \\
\hline Donepezil $10 \mathrm{mg}$ & 0.01 & 0.12 & 4.74 & 1.3 \\
\hline Memantine & 0.16 & 0.36 & 3.34 & 1.65 \\
\hline Quetiapine & 0.06 & 0.24 & 5.27 & 1.8 \\
\hline Rivastigmine & 0.02 & 0.13 & 6.2 & 1.33 \\
\hline \multicolumn{5}{|l|}{ Discontinuation rate } \\
\hline Placebo & 0.01 & 0.09 & 4.02 & 1.11 \\
\hline Donepezil $3 \mathrm{mg}$ & 0.1 & 0.3 & 4.19 & 1.95 \\
\hline Donepezil $5 \mathrm{mg}$ & 0.01 & 0.09 & 5.53 & 1.3 \\
\hline Donepezil $10 \mathrm{mg}$ & 0.09 & 0.29 & 3.14 & 1.4 \\
\hline Memantine & 0.11 & 0.32 & 3.37 & 1.69 \\
\hline Quetiapine & 0.65 & 0.48 & 1.91 & 1.59 \\
\hline Rivastigmine & 0.03 & 0.16 & 5.85 & 1.63 \\
\hline \multicolumn{5}{|c|}{ Discontinuation rate due to AEs } \\
\hline Placebo & 0.01 & 0.07 & 4.34 & 1.18 \\
\hline Donepezil $3 \mathrm{mg}$ & 0.09 & 0.29 & 4.12 & 1.93 \\
\hline Donepezil $5 \mathrm{mg}$ & 0.01 & 0.1 & 5.22 & 1.48 \\
\hline Donepezil $10 \mathrm{mg}$ & 0.41 & 0.49 & 2.02 & 1.18 \\
\hline Memantine & 0.08 & 0.27 & 3.93 & 1.75 \\
\hline Quetiapine & 0.31 & 0.46 & 4.02 & 2.58 \\
\hline Rivastigmine & 0.09 & 0.29 & 4.35 & 1.97 \\
\hline \multicolumn{5}{|l|}{ Psychiatric events } \\
\hline Placebo & 0.07 & 0.26 & 2.93 & 1.05 \\
\hline Donepezil $3 \mathrm{mg}$ & 0.03 & 0.17 & 4.82 & 1.32 \\
\hline Donepezil $5 \mathrm{mg}$ & 0.04 & 0.21 & 3.65 & 1.22 \\
\hline
\end{tabular}

Table 3 continued

\begin{tabular}{|c|c|c|c|c|}
\hline \multirow[t]{2}{*}{$\begin{array}{l}\text { Treatment option/and } \\
\text { efficacy/safety outcome }\end{array}$} & \multicolumn{2}{|c|}{$\begin{array}{l}\text { Probability } \\
\text { of being the } \\
\text { safest } \\
\text { treatment }\end{array}$} & \multicolumn{2}{|c|}{$\begin{array}{l}\text { Rank of } \\
\text { treatment }\end{array}$} \\
\hline & Mean & SD & Mean & SD \\
\hline Donepezil $10 \mathrm{mg}$ & 0.39 & 0.49 & 2.06 & 1.12 \\
\hline Memantine & 0.41 & 0.49 & 2.7 & 1.77 \\
\hline Quetiapine & 0.05 & 0.22 & 4.85 & 1.46 \\
\hline
\end{tabular}

\section{Efficacy Outcome Measures of the NMA}

The median treatment effect and confidence interval for each efficacy outcome comparison of each active treatment versus placebo is shown in Fig. 1. Table 2 includes the likelihood of each treatment option being the best and the average rank of each treatment option for each efficacy measure.

For the six RCTs assessing cognitive function using MMSE, baseline mean values ranged from 17.2 to 20.6 across each arm of the included trials, indicating mild to moderate cognitive impairment. Time points used in the NMA for assessing change from baseline ranged from 12 to 24 weeks. For one study with a 16 -week placebo-controlled phase followed by an open-label switch phase [12], the MMSE score was taken from the 16-week time point. In this study, donepezil $5 \mathrm{mg}$ was the most effective treatment compared to placebo based on increase in mean MMSE from baseline, although the difference was non-significant. This was followed by donepezil $10 \mathrm{mg}$, rivastigmine and donepezil $3 \mathrm{mg}$. Both memantine and quetiapine showed a non-significant change in mean MMSE score from baseline favouring placebo [12].

Four studies assessed neuropsychiatric symptoms using mean scores of the NPI-10, the most commonly reported variant of NPI. Baseline mean scores ranged from 16.6 to 23.2 across each arm of the included trials, and the time points used for assessing change from baseline 
ranged from 12 to 24 weeks following randomisation. Donepezil $10 \mathrm{mg}$ and rivastigmine were non-significantly the most effective treatments for reducing mean NPI-10 scores. Other treatments had a mean and median treatment effect close to zero with wide confidence intervals favouring placebo.

In terms of improvement in motor function, including extrapyramidal symptoms, four studies assessed the reduction from baseline in mean UPDRS Part III score and one study each reported on mean Part II or modified UPDRS scores. Time points used for assessing change from baseline ranged from 10 to 24 weeks. Donepezil $5 \mathrm{mg}$, donepezil $3 \mathrm{mg}$ and, to a lesser degree, memantine were identified as effective treatments for reducing UPDRS component scores. These treatments effects, however, were not statistically significant. Compared to placebo, quetiapine and donepezil $10 \mathrm{mg}$ showed slight non-significant increases in scores from baseline.

Sensitivity analyses conducted for each efficacy outcome measure closely aligned with the base-case results. In the FE model for MMSE, differences versus placebo for donepezil $5 \mathrm{mg}$, donepezil $10 \mathrm{mg}$ and memantine were statistically significant.

\section{Safety Outcome Measures of the NMA}

The odds ratio for each safety outcome comparison of each treatment versus placebo is presented in Fig. 2. Table 3 includes the likelihood of each treatment option having the lowest risk of each safety event and the average rank of each treatment option for each safety measure.

Eight RCTs provided data on AEs and discontinuations over a study period ranging between 10 and 24 weeks, with the exception of the study of Mori et al. [12] which recorded AEs up to 52 weeks. One study each did not report overall AEs [27] or total discontinuation rates [20]. Data on psychiatric events were only available from five studies.

Donepezil $3 \mathrm{mg}$ was associated with a lower odds ratio for overall AEs compared to placebo; while all other treatments had higher risk, with the highest being rivastigmine followed by quetiapine. Dose-dependent increases in odds ratio for overall AEs were observed for donepezil. However, differences compared to placebo for overall AEs were not significant.

The risk of overall discontinuation was lower for donepezil $10 \mathrm{mg}$, memantine and quetiapine compared to placebo. For other treatments, the risk of overall discontinuation was higher than that of placebo, with the highest risk being for rivastigmine. This result was supported by similar results for discontinuation due to AEs. Differences between placebo and all treatments in relation to overall discontinuation and discontinuation due to AEs were not statistically significant.

Donepezil $10 \mathrm{mg}$, followed by memantine, had the lowest risk for psychiatric AEs, with the risk being lower than that for placebo, although the difference was non-significant.

\section{DISCUSSION}

To our knowledge, this study is the first NMA that has been developed to provide a comparative analysis across the available therapy options for DLB. Given that all of the included trials were conducted compared to placebo, NMA makes it possible to combine all of the available placebo-controlled trials so that the included existing treatment options for DLB can be indirectly compared.

The findings that donepezil and rivastigmine show the best efficacy profiles is consistent with and supports current clinical practice favouring the use of acetylcholinesterase inhibitors as first-line pharmacological treatment for patients with DLB [28]. Evidence suggesting that the cholinergic hypothesis fits better with DLB than AD provides additional rationale for the use of these medicines, which have been licensed for the treatment of AD [29]. Based on the NPI measurement scale, there was a high probability of rivastigmine being the best performing treatment in terms of efficacy alone. For donepezil, the effect is greater at the $5 \mathrm{mg}$ and $10 \mathrm{mg}$ doses. It may be expected that different dose amounts and formulations available for rivastigmine would produce varying results, 
as seen for donepezil; however, the available trial data did not differentiate between these within the available publications focusing on DLB patients.

In terms of safety, rivastigmine and quetiapine appear to rank the lowest in the safety/tolerability profile. For rivastigmine, gastrointestinal side effects, hypersalivation, lacrimation and urinary frequency may likely be the predominant reason for higher rates of discontinuation compared to placebo in its clinical trials. Such side effects, which increase patient self-care and carer burden, may indeed be more troublesome in patients with DLB; however, this notion is not borne out in the NMA, with discontinuation rates due to AEs compared to placebo being comparable-albeit with extremely wide confidence intervals (OR 1.04; 95\% CI 0.04, 27.71).

For quetiapine, which was investigated specifically for managing agitation and psychosis in patients with dementia, higher odds of AEs (OR 3.73; 95\% CI 0.69, 21.49) do not lead to higher odds of discontinuation or discontinuations due to AEs versus placebo (OR 0.29; 95\% CI 0.02, 3.43 and OR 0.85; 95\% CI 0.00, 901.8, respectively). However, regulatory bodies have highlighted caution with their use in older patients with dementia-related psychosis due to increased risk of cerebrovascular accidents and death [17]. Additionally, there are strong clinical recommendations to avoid the use of antipsychotics in older patients due to an associated greater rate of cognitive decline [30]. In relation to DLB specifically, severe sensitivity reactions, including exacerbation of parkinsonism, confusion and autonomic dysfunction. may particularly limit the usefulness of antipsychotics. Furthermore, greater cognitive decline and confusion may lead to further psychiatric events, which do appear to be increased for quetiapine versus placebo (OR 2.53; 95\% CI $0.35,20.88$ ) and all other comparators (see section Safety Outcome Measures of the NMA).

The mixed findings reported to date for memantine are reinforced in this NMA. While in clinical trials memantine demonstrated a greater magnitude of clinical global impression of change than other agents [18], based on the present analysis, it appears to perform less well on secondary measures, such as those explored in this NMA. For the MMSE measurement scale, the outcomes even suggest that, at the mean level, memantine performed worse than placebo. This in part captures the high levels of uncertainty in the MMSE outcome, where the confidence interval crosses the point of zero difference compared to placebo. Additionally, if memantine is treating symptoms not captured within this measure, but the AEs have an impact on cognitive functions, there may appear to be a worsening compared to placebo in this one measurement scale. This possibility further highlights the need to compare the treatments based on a range of available metrics and that the decision should not be based on a single metric when selecting an appropriate treatment option for each patient. In terms of safety, overall AEs were found to be lower for memantine compared with donepezil $10 \mathrm{mg}$ (OR 0.98; 95\% CI 0.24, 19.36). Discontinuation rates due to AEs were higher for memantine (OR 2.95 ; $95 \%$ CI $0.15,79.70$ ), which may be due to increased psychiatric events (OR 1.11; 95\% CI $0.16,75.67)$. The relationship found here between discontinuations and psychiatric events is consistent with the increased risk of delusions and hallucinations that may be particularly problematic for patients with DLB.

Donepezil $3 \mathrm{mg}$ had lower rates of AEs but higher rates of overall discontinuation and discontinuation due to AEs compared with the $5 \mathrm{mg}$ and $10 \mathrm{mg}$ doses. This could possibly be explained by greater efficacy at higher doses, potentially leading to patients and clinicians having an increased willingness to tolerate AEs. In terms of donepezil $3 \mathrm{mg}$, these findings support the current Japanese license in which this is only an initial dose, with tolerability-based up-titration to $5 \mathrm{mg}$ and $10 \mathrm{mg}$ recommended for the treatment of DLB [10].

Overall, while rivastigmine performed highly in terms of the efficacy, its safety outcomes were less favourable. Donepezil $5 \mathrm{mg}$ ranked consistently higher on the efficacy and safety outcomes compared to other treatments. This network of evidence therefore suggests that donepezil $5 \mathrm{mg}$ may have the best overall risk/ benefit profile for the treatment of patients with DLB. Indeed, in real-world clinical assessments 
of patients, there may a potential for a better selection of those who may derive the greatest benefit from donepezil $10 \mathrm{mg}$, based on overall benefit/risk for particular individuals.

Although these trends were present in the estimated values, there were no statistically significant results observed. The lack of significance may be driven either by the limited availability of data to inform the analysis, or by heterogeneity within the study designs and populations. A high level of heterogeneity within each of the study populations may suggest that a single preferred treatment option to use across all DLB patients may not be preferable. Instead, it may suggest that the treatment option provided should be tailored to each individual, leading to the approach of personalised treatments for patients. The difference between a traditional shrewd assessment of a complex medical condition and a true personalised (or precision) medicine is the degree of reliance on data to objectively make decisions about specific treatment paths that may be more effective for each individual. Given the nature and quality of the currently available data presented here, it is difficult to achieve.

Two specific sensitivity analyses, one pooling all doses of donepezil and another excluding mixed populations, did not impact the trends seen in the base-case scenario or their significance. Additionally, the impact on heterogeneity in the sensitivity analyses was inconsistent across different outcome measures.

In the pooled donepezil dose analyses, the lack of change could be explained by the fact that the $3 \mathrm{mg}$ dose is only an initiation dose and effectiveness would be best assessed when individuals are maintained for adequate time on the maximum tolerated dose of either $5 \mathrm{mg}$ or $10 \mathrm{mg}$. This would also be more in keeping with actual clinical practice, where the aim is to optimise effectiveness while balancing tolerability, as well as being a better indirect comparison with other drugs for which up-titration is a standard protocol.

Due to the exclusion of studies, there were no data to include quetiapine in the analysis excluding mixed populations. For the other comparators, the lack of difference in the mixed populations exclusion analysis could be partly explained by the relatively small proportions of patients with Pervasive Developmental Disorder (PDD) or AD with parkinsonian features in the studies that formed the base-case NMA. This provides reassurance, as while there may be differences in the preponderance of specific symptoms across DLB, PDD and AD with parkinsonian features, reaching a clear diagnosis may be challenging in many patients.

\section{Study Limitations}

There are a number of major limitations to the study.

First, the UPDRS was used to confirm diagnosis based on the presence of parkinsonian features; therefore, the NMA protocol treated this outcome as an efficacy measure. However, parkinsonian features could worsen as a result of drug exposure, so UPDRS may also be considered as a safety measure. As such, no active drug significantly worsened parkinsonian symptoms/signs compared to placebo. It is worth noting that the rivastigmine studies did not use the UPDRS as an assessment instrument.

Second, while common trends support clinical effectiveness beyond random variations, actual differences between comparators were not statistically significant, confidence intervals were wide and a high degree of heterogeneity was present.

Third, the small number of clinical trials, with the majority only directly comparing with placebo, and the relatively small number of patients with DLB exposed to specific pharmacological interventions may contribute to the lack of statistical significance and broad confidence intervals leading to uncertainty. This highlights the need for further research, with studies comparing treatments to active comparators and utilising outcome measures that may be more sensitive and specific to DLB.

Fourth, limited data were provided in each of the studies to allow for adjustments to limit the impact of effects on the outcome measures beyond those from the active treatments. Missing or limited data may include factors such as education levels or the use of 
concomitant L-dopa dosing or L-dopa equivalents. Evidence does suggest that L-dopa does not compromise the cognitive or behavioural profiles of patients with DLB, either acutely or over a longer period of time, and would therefore have a limited impact on the included measures [16].

Finally, the low number of trials included for each outcome restricted the options available for more advanced analytical techniques, such as meta-regression. With additional data availability, a meta-regression would be able to control for a range of factors which may introduce heterogeneity and produce results that may adjust for the underlying patient characteristics.

\section{CONCLUSION}

As the interventions explored in this NMA have different modes of action, it is possible that standardised instruments are not sensitive to improvements in specific symptoms that may be better controlled by specific drugs and may be of particular concern for individual patients. Therefore, in the absence of a disease-modifying treatment, general management should continue to be symptomatic, with a focus on balancing benefit/risk for individual patients.

Given the low number of trials and the high degree of heterogeneity between the studies included in this NMA, caution should be exercised in making decisions based upon the results produced by the NMA. The results presented from this NMA should be used to explore the expected trends in the data, rather than as a substitute for direct evidence for the purposes of economic modelling or other analyses. It is likely that additional evidence would have a high impact on the results, particularly if headto-head evidence was introduced to the network. This highlights the need for further research with studies comparing treatments to active comparators and utilising measures that may be more sensitive and specific to DLB.

Alongside the limitations of the available data, the lack of significant results across each of the included measures may also support the need for a more personalised treatment approach for DLB patients. Due to the heterogeneity across the range of patients who may be characterised as suffering from DLB, personalised medicine needs to be based on the different characteristics of individual patients. By combining clinical, pharmaceutical and socioeconomic information, one may observe patterns in the effectiveness of particular treatments and identify the characteristics, such as genetic, environmental and lifestyle variations, that may be correlated with success or failure for each treatment option.

In conclusion, this is the first NMA exploring the comparative efficacy and safety of pharmacological interventions used for the treatment of patients with DLB. While the results are not statistically significant, donepezil $5 \mathrm{mg}$ ranked highest on the efficacy and safety outcomes; it may result in the best overall benefit/risk profile for the treatment of patients with DLB. Future trials should aim to undertake direct head-tohead treatment comparisons to improve the evidence base for a disease with a high unmet need.

\section{ACKNOWLEDGEMENTS}

The authors thank Genevieve Meier, employee of Eisai, for her input to the design of both the systematic literature review and NMA on which this manuscript reports.

Funding. This research was funded by Eisai. Adelphi Values received funding from Eisai to support this research. The Journal's Rapid Service and Open Access Fees was also funded by Eisai.

Authorship. All named authors meet the International Committee of Medical Journal Editors (ICMJE) criteria for authorship for this manuscript, take responsibility for the integrity of the work as a whole, and have given final approval to the version to be published. All authors had full access to the articles reviewed in this manuscript and take complete responsibility for the integrity and accuracy of this 
manuscript. Amir A. Tahami Monfared serves as overall guarantor for this study and manuscript.

Authorship Contributions. Amir A. Tahami Monfared and Richard Perry conceived the study and were in charge of overall direction and planning and provided critical feedback to the other authors. Stefano Lucherini, Robert Hughes and Yunni Yi provided support to the design and the analysis of data, and interpretation of the results. Mitesh Desai provided critical oversight of the analysis and interpretation of the results.

Disclosures. Eisai employed Amir A. Tahami Monfared, who played a significant part in study design, data collection, data analysis, data interpretation and writing of the report. Mitesh Desai, Stefano Lucherini, Robert Hughes, Yunni Yi and Richard Perry have nothing to disclose.

Compliance with Ethics Guidelines. This article is based on previously conducted studies and does not contain any studies with human participants or animals performed by any of the authors.

Data Availability. The datasets used and/or analysed during the current study are available from the corresponding author, Amir A. Tahami Monafred, on reasonable request.

Open Access. This article is licensed under a Creative Commons Attribution-NonCommercial 4.0 International License, which permits any non-commercial use, sharing, adaptation, distribution and reproduction in any medium or format, as long as you give appropriate credit to the original author(s) and the source, provide a link to the Creative Commons licence, and indicate if changes were made. The images or other third party material in this article are included in the article's Creative Commons licence, unless indicated otherwise in a credit line to the material. If material is not included in the article's Creative Commons licence and your intended use is not permitted by statutory regulation or exceeds the permitted use, you will need to obtain permission directly from the copyright holder. To view a copy of this licence, visit http://creativecommons.org/licenses/bync/4.0/.

\section{REFERENCES}

1. Alzheimer's Research UK. Types of dementia. Dementia information Web site. 2019. https:// www.alzheimersresearchuk.org/dementiainformation/types-of-dementia/dementia-withlewy-bodies/. Accessed 11 May 2020.

2. Zaccai J, McCracken C, Brayne C. A systematic review of prevalence and incidence studies of dementia with Lewy bodies. Age Ageing. 2005;34(6):561-6.

3. Mrak RE, Griffin WST. Dementia with Lewy bodies: definition, diagnosis, and pathogenic relationship to Alzheimer's disease. Neuropsychiatr Disease Treat. 2007;3(5):619.

4. McKeith IG, Boeve BF, Dickson DW, et al. Diagnosis and management of dementia with Lewy bodies: fourth consensus report of the DLB Consortium. Neurology. 2017;89(1):88100.

5. Mueller C, Ballard C, Corbett A, Aarsland D. The prognosis of dementia with Lewy bodies. Lancet Neurol. 2017;16(5):390-8.

6. Bharmal MF, Dedhiya S, Craig BA, et al. Incremental dementia-related expenditures in a medicaid population. Am J Geriatric Psychiatry. 2012;20(1): 73-83.

7. Kronborg Andersen C, Lauridsen J, Andersen K, Kragh-Sørensen P. Cost of dementia: impact of disease progression estimated in longitudinal data. Scand J Public Health. 2003;31(2):119-25.

8. O'Brien JT, Burns A. Clinical practice with anti-dementia drugs: a revised (second) consensus statement from the British Association for Psychopharmacology. J Psychopharmacol. 2011;25(8):997-1019.

9. Mori E, Ikeda M, Nakai K, Miyagishi H, Nakagawa $\mathrm{M}$, Kosaka K. Increased plasma donepezil concentration improves cognitive function in patients with dementia with Lewy bodies: an exploratory pharmacokinetic/pharmacodynamic analysis in a phase 3 randomized controlled trial. J Neurol Sci. 2016;366:184-90.

10. Eisai Co. ARICEPT $\AA$ approved in Japan as treatment for dementia with Lewy bodies. 2014. https://www. eisai.com/news/news201452.html. Accessed Jan 2020. 
11. Ikeda M, Mori E, Matsuo K, Nakagawa M, Kosaka K. Donepezil for dementia with Lewy bodies: a randomized, placebo-controlled, confirmatory phase III trial. Alzheimer's Res Therapy. 2015;7(1):4.

12. Mori E, Ikeda M, Nagai R, Matsuo K, Nakagawa M, Kosaka K. Long-term donepezil use for dementia with Lewy bodies: results from an open-label extension of Phase III trial. Alzheimer's Res Therapy. $2015 ; 7(1): 5$.

13. Tahami Monfared AA, Meier G, Perry R, Joe D. Burden of disease and current management of dementia with Lewy bodies: a literature review. Neurol Therapy. 2019;8(2):289-305.

14. Shamseer L, Moher D, Clarke M, et al. Preferred reporting items for systematic review and metaanalysis protocols (PRISMA-P) 2015: elaboration and explanation. BMJ. 2015;349:g7647.

15. Hutton B, Salanti G, Caldwell DM, et al. The PRISMA extension statement for reporting of systematic reviews incorporating network meta-analyses of health care interventions: checklist and explanations. Ann Intern Med. 2015;162(11): 777-84.

16. Molloy S, Rowan E, O'brien J, McKeith I, Wesnes K, Burn D. Effect of levodopa on cognitive function in Parkinson's disease with and without dementia and dementia with Lewy bodies. J Neurol Neurosurg Psychiatry. 2006;77(12):1323-8.

17. US Food and Drug Administration (FDA). Aricept (donepezil hydrochloride) label. FDA product labelling web site. 2019. https://www.accessdata. fda.gov/drugsatfda_docs/label/2012/020690s035,02 1720s008,022568s005lbl.pdf. Accessed Sept 2019.

18. Aarsland D, Ballard C, Walker Z, et al. Memantine in patients with Parkinson's disease dementia or dementia with Lewy bodies: a double-blind, placebo-controlled, multicentre trial. Lancet Neurol. 2009;8(7):613-8.

19. Culo S, Mulsant BH, Rosen J, et al. Treating neuropsychiatric symptoms in dementia with Lewy bodies: a randomized controlled-trial. Alzheimer Dis Assoc Disord. 2010;24(4):360-4.

20. Kurlan R, Cummings J, Raman R, Thal L. Quetiapine for agitation or psychosis in patients with dementia and parkinsonism. Neurology. 2007;68(17):1356-63.
21. McKeith I, Del Ser T, Spano P, et al. Efficacy of rivastigmine in dementia with Lewy bodies: a randomised, double-blind, placebo-controlled international study. Lancet. 2000;356(9247):2031-6.

22. Emre M, Tsolaki M, Bonuccelli U, et al. Memantine for patients with Parkinson's disease dementia or dementia with Lewy bodies: a randomised, doubleblind, placebo-controlled trial. Lancet Neurol. 2010;9(10):969-77.

23. Mori E, Ikeda M, Kosaka K, Donepezil DLBDI. Donepezil for dementia with Lewy bodies: a randomized, placebo-controlled trial. Ann Neurol. 2012;72(1):41-52.

24. Cummings JL, Street J, Masterman D, Clark WS. Efficacy of olanzapine in the treatment of psychosis in dementia with Lewy bodies. Dement Geriatr Cogn Disord. 2002;13(2):67-73.

25. Goetz CG. Unified Parkinson's Disease Rating Scale (UPDRS) and The Movement-Disorder Society Sponsored-unified Parkinson's Disease Rating Scale (MDS-UPDRS). In: Kompoliti K, Metman LV, editors. Encyclopedia of movement disorders. Oxford: Academic Press; 2010. p. 307-309.

26. Dias S, Welton NJ, Sutton AJ, Ades A. NICE DSU technical support document 2: a generalised linear modelling framework for pairwise and network meta-analysis of randomised controlled trials. 2011. http://www.nicedsu.org.uk/TSD2\%20General\% 20meta\%20analysis\%20corrected\%20Mar2013.pdf. Accessed Jan 2020.

27. Wesnes KA, McKeith IG, Ferrara R, et al. Effects of rivastigmine on cognitive function in dementia with lewy bodies: a randomised placebo-controlled international study using the cognitive drug research computerised assessment system. Dement Geriatr Cogn Disord. 2002;13(3):183-92.

28. Lewy Body Dementia Association. Treatment options. Learn about LBD web site. 2016. https:// www.lbda.org/go/treatment-0. Accessed Jan 2020.

29. Ferman TJ, Boeve BF. Dementia with Lewy bodies. Neurol Clin. 2007;25(3):741-60.

30. Panel AGSBCUE, Fick DM, Semla TP, et al. American Geriatrics Society 2019 updated AGS Beers Criteria ${ }^{\circledR}$ for potentially inappropriate medication use in older adults. J Am Geriatr Soc. 2019;67(4): 674-94. 